www. revis ta d y o. com

\title{
La Nueva Cadena de Suministro en la Era de los E-Retailers: Una Revisión Bibliográfica Actualizada
}

\author{
The New Supply Chain in the Era of E-Retailers: A State-of-the-art Literature Review
}

Rodríguez-García, M., Domínguez-Caamaño, P. y Prado-Prado, J. C.

Grupo de Ingeniería de Organización. Dpto. de Organización de Empresas y Marketing. Escuela de Ingeniería Industrial. Universidad de Vigo. c/Maxwell, s/n - 36310 Vigo, España

miguelgarcia@uvigo.es; padominguez@uvigo.esy jcprado@uvigo.es

Fecha de recepción: 27-06-2016

Fecha de aceptación: 19-07-2016

Resumen El crecimiento global del comercio electrónico continúa y las cadenas de suministro tradicionales se reinventan. A día de hoy, todavía no existe un marco que defina qué temas han sido los más presentes en la literatura sobre este aspecto. El objetivo de este artículo es ofrecer una recopilación bibliográfica actualizada sobre las implicaciones de este nuevo canal de ventas en la cadena de suministro, con una visión centrada en la figura de los e-retailers. En este marco, analizamos tanto los modelos de venta que se centran únicamente en el mercado online (puros e-retailers), como los que desarrollan una estrategia multicanal.

Palabras clave: comercio electrónico, multicanal, e-retailer, cadena de suministro, revisión bibliográfica

\begin{abstract}
E-commerce has been growing tremendously over the past few years, and traditional supply chains are now compelled to reinvent themselves. An analysis of the literature shows that a frame for the most common topics on this new environment is not adequately developed. The main goal of this paper is to develop a state-of-the-art literature review on the implications of the online channel for traditional supply chains, and to identify possible gaps between available literature and real management issues.
\end{abstract}

The literature review is based on 62 journal articles published between 2011 and 2016, and all the papers are classified according to the main themes addressed and the research methodology. This establishes a new frame-work that helps academics, researchers, and practitioners better understand new types of sup-ply chains and inspire future research. The publications were analyzed from a retailer perspective; there-fore the business-to-consumer (B2C) sales mode is the main focus of our review. Business-to-business (B2B) oriented publications were not included in the analysis. Also, we focus our analysis on both pure e-retailers and multi-channel retailers. Pure e-retailers sell products only through the online channel, and multi-channel retailers operate through both traditional and online channels. This integration between channels brings new opportunities for traditional brick-and-mortar retailers, but it also requires a lot of trade-off decisions. Market cannibalization, which refers to a decrease in retail sales as a result of the in-troduction of the online channel, is one of the biggest challenges companies face. Furthermore, with the rise of e-commerce, marketing and operations management are more closely interlinked, and therefore decisions in both fields are included in this review.

We identify e-commerce adoption and network design, customer service, pricing, e-fulfillment, inventory management, reverse logistics, and environmental issues as the main topics regarding new supply chains. As far as the methodology is concerned, mathematical models and empirical studies are the most common research methodology methods, and combined they represent more than $80 \%$ of the sample of publications.

Keywords: e-commerce, multi-channel, e-retailer, supply chain, literature review 


\section{Introducción}

Según datos publicados por la consultora A. T. Kearney, las ventas globales en el mundo del comercio electrónico continúan creciendo a ritmos imparables. Con un aumento medio cercano al $20 \%$ anual en los últimos 3 años (2012-2015), el total de ventas online en el año 2015 alcanzó los $\$ 994.5$ billones de dólares americanos, y se espera que para el año 2018 está cifra sobrepase los $\$ 1,500$ billones (A.T. Kearney, 2015).

Esta venta directa al cliente desde el canal online es comúnmente conocida por los términos en inglés e-commerce o B2C (Business to Costumer), mientras que existen otras modalidades en el comercio electrónico como el B2B (Business to Business), centrado en la venta de productos entre empresas. El concepto de e-retailer, utilizado inicialmente para hacer referencia a las empresas que exclusivamente operan online, dada la integración cada vez mayor entre ambos canales, online y offline, se aplica también a compañías que operan a través ambos canales.

La rápida expansión de las tecnologías de la comunicación en los últimos años ha permitido a numerosas empresas retailers del mundo ampliar sus cartera de contactos y sus ventas de forma masiva gracias a los nuevos clientes del canal online y convertirse en e-retailers (Setia et al., 2013; Hunter, 2015). Cada vez más y más nuevas compañías deciden basar su actividad principal en el negocio online, mientras que aquellas ya existentes y con una fuerte presencia en el mercado tradicional se ven también obligadas a adoptar estrategias multicanal que le permitan ser competitivas en el mercado. Dos ejemplos que reflejan ambas tendencias son Amazon, líder del mercado de los e-retailers y con una estrategia únicamente centrada en las ventas online, mientras que Wal-Mart (el mayor retailer del mundo), se sitúa en segunda posición del mercado online con un modelo integrador de la venta física y virtual (Wahba, 2016).

Ante este nuevo entorno de oportunidades y grandes cambios que presentan la venta on-line y las estrategias multicanal, las cadenas de suministro se reinventan, y esto incluye decisiones clave tanto en el área de marketing como en el área de la logística.

Desde un punto de vista del marketing, Internet es una de las herramientas más poderosas proveyendo información al consumidor, reduciendo en gran medida los costes de búsqueda de los clientes. Los consumidores tienen la posibilidad de comparar múltiples ofertas con gran facilidad, lo que reduce la fidelidad de los clientes e incrementa la presión competitiva sobre todas las empresas (Li et al., 2015). Esta situación, junto con la creciente demanda global y los ciclos de vida de producto cada vez más cortos, fuerza a las compañías a reducir drásticamente sus costes para poder competir en precios, a la vez que deben de aumentar su cartera de productos y servicios para así dar más alicientes a los clientes para elegir sus webs de venta (Stritto y Schiraldi, 2013). Más y más consumidores se están volviendo compradores multicanal, por lo que los conflictos y la canibalización entre canales pueden ser consecuencias directas de una mala estrategia.

Desde el punto de vista logístico, la venta online implica nuevos retos muy importantes debido al mayor nivel de servicio que demandan los clientes. Por un lado, la distribución es un punto crítico, con tiempos de entrega cada vez más cortos y con múltiples destinos a los que llegar con nuestros productos (Cleophas y Ehmke, 2014). Además, el creciente número de referencias a manejar, consecuencia del marketing online, tiene un impacto tremendo en la logística, que debe procesar y distribuir todos esos productos. Por otro lado, la integración de los canales presenta nuevas oportunidades para las empresas tradicionales. Un ejemplo es el cambio de enfoque para una empresa como Wal-Mart, pasando de un modelo de grandes envíos desde las plataformas logísticas hacia las tiendas, a otro modelo que busca entregas individuales al cliente final. Alternativas en los modelos de venta como el pick-up at store (compra online - recoge en tienda) posibilitan la integración de muchos procesos logísticos que los hace más eficientes (Gallino y Moreno, 2014).

El conjunto de nuevos retos que supone el e-commerce para las empresas se pone de manifiesto en la creciente cantidad de publicaciones existente en la literatura, hecho que se refleja en la muestra tomada para esta revisión y que se detalla más adelante. Por ello, una recopilación bibliográfica actualizada de los últimos años se muestra de gran utilidad. El enfoque de nuestro trabajo busca presentar todas estas nuevas tendencias de publicación que definen los cambios en la cadena de suministro tradicional, con una visión centrada principalmente en la figura de los e-retailers y la venta directa al consu-midor (B2C).

\section{Metodología}

En esta sección, se presenta la metodología seguida por los autores para el análisis de la literatura existente. El desarrollo de la investigación se llevó a cabo de forma estructurada, tomando como base las etapas que Seuring y Müller (2008) utilizaron en su proceso de revisión bibliográfica. 
1. Acotación del marco de búsqueda y de las categorías de análisis.

2. Determinación de los parámetros de búsqueda para cada categoría.

3. Análisis del material encontrado.

4. Selección del material que se incluirá finalmente en la revisión bibliográfica.

\section{Preparación de los resultados.}

6. Análisis del feedback extraído de los resultados y redefinición del proceso de búsqueda si es necesario.

El marco de búsqueda inicial se definió en base al objetivo principal de los autores, que buscan presentar una revisión actualizada de la literatura sobre las principales temáticas que afectan a las nuevas cadenas de suministro como consecuencia de la aparición y despunte del canal de venta online en el mercado, y centrándose en el punto de vista los e-retailers. En esta primera fase, todavía no se habían definido los grupos de temáticas que iban a englobar a las publicaciones analizadas. Cada una de las subcategorías fue definida posteriormente en base a los resultados que se obtenían en el proceso de investigación. En cada una de ellas, se incluyen tanto aquellos estudios que se centran únicamente en la venta online como aquellos que aportan ciertos conocimientos sobre la gestión multicanal.

Además, el estudio también incorpora otro análisis complementario sobre el tipo de publicaciones, clasificadas en función de la metodología de estudio de las mismas. Cinco categorías fueron definidas: Teórico, Case-Study, Empírico, Modelo y Revisión Bi-bliográfica (Seuring y Müller, 2008).

Entre los términos clave utilizados en la búsqueda se pueden destacar: "e-commerce", "online sales", "retail", "retailer", "e-retailer", "suppy chain", "logistics", "B2C", "multi-channel" y otros más concretos para cada uno de los grupos de publicaciones definidos, que se incorporaron a la búsqueda tras analizar las principales tendencias de la primera muestra de publicaciones. Ejemplos de términos clave que se incorporaron a posteriori son "picking", "third-party logistics" o "pick-up at store", todos ellos ampliamente utilizados en el mundo de las ventas online.

El proceso de búsqueda se llevó a cabo en la base de datos Scopus y el marco temporal de nuestra revisión engloba todas las publicaciones publicadas entre los años 2011 y 2016, ambos incluidos.

La unidad de análisis fue única, ya que nuestra revisión incluye solamente artículos de revistas, lo cual consideramos que ayuda a homogeneizar el estudio y a darle un carácter más riguroso. Además, el único idioma contemplado en el proceso de revisión fue el inglés.

Una revisión empírica de cada una de las publicaciones dejó fuera del estudio aquellas que no estaban realmente enfocadas al objeto de estudio. Un ejemplo de esto son aquellas publicaciones centradas en el comercio electrónico B2B o en el B2C del sector servicios. Lo mismo se aplica a canales de venta de servicios de cualquier tipo, ya que nos limitamos únicamente a la venta de productos físicos. Además, de entre estas publicaciones, aquellas que presentaban casos muy específicos, como las centradas en el estudio de una única compañía o con conclusiones sólo aplicables a áreas geográficas reducidas, también fueron excluidas del análisis.

En total, más de 100 artículos han sido analizados, para finalmente conformar una muestra de 62 publicaciones. Se muestran otras 6 referencias que no se incluyeron en el estudio, pero que igualmente aparecen referenciadas (para un total de 68 referencias), y que ayudan a dar forma y completar el artículo.

\section{Resultados}

Las publicaciones se han agrupado de manera heurística en base a su contenido en 7 grandes grupos. El objetivo de esta clasificación es facilitar el entendimiento de las numerosas problemáticas que se tratan en la literatura a través de un esquema sencillo. Estos grupos no se deben considerar rígidos y, en realidad, las temáticas que abarcan cada que uno de ellos están fuertemente interrelacionadas. Por esta razón, muchas de las pu-blicaciones se podrían asignar a más de uno ellos. La asignación final de un artículo a un grupo u otro se ha hecho tratando de determinar cual era la aportación principal del mismo. A continuación, se describen cada uno de los grupos definidos:

- Adopción y diseño del canal online: la aparición del canal online continúa generando discusión años después de su popularización a comienzo de los años 2000. La introducción de las ventas online ha sido una gran oportunidad de negocio para algunas empresas, dándoles acceso a clientes a los que antes no lo tenían. Sin embargo, para otros ha sido un gran reto por lo que implica introducirse en este nuevo canal de ventas. Por ello, muchas de las publicaciones ha- 
cen referencia a las consideraciones tanto en el diseño como en la integración de este nuevo canal con el canal tradicional.

- Precios: el comportamiento de los precios es uno de los elementos clave en cualquier negocio, pero quizás más aún en un mercado online en donde la competencia es global y muy extensa. Como tal, tiene una fuerte representación en este análisis bibliográfico ya que numerosos autores se centraron en este aspecto.

Servicio al cliente: otro grupo de publicaciones enumeran los factores que afectan al servicio percibido por los clientes en el comercio electrónico, entre los que destacan tantos los procesos de transacción online, en los que se incluyen la calidad de la web o las opciones de pago para el cliente, como los servicios logísticos ofrecidos: plazo, flexibilidad en la entrega, gestión de las devoluciones, etc.

- $\quad$ E-fulfillment: es sin lugar a dudas el área de operaciones más estudiada del comercio electrónico. El e-fulfillment se entiende como aquel conjunto de procesos necesarios para hacer llegar el producto al cliente desde que se produce la venta. Dentro de este grupo se pueden incluir las operaciones de preparación de pedidos, el envío al cliente y la venta misma.

- Gestión de stocks: el combinar dos modelos de venta distintos como el online y offline ha abierto una nueva puerta a la gestión integrada de stocks entre ambos canales. Muchos autores describen mod- elos que buscan reducir los stocks totales gracias a la centralización.

- Devoluciones y logística inversa: el estudio de la logística inversa tiene gran importancia en el comercio electrónico, debido principalmente a la dificultad para los e-retailers de vender grandes cantidades de productos online sin tener que manejar una gran cantidad de productos devueltos por los consumidores. Al ser un canal que fomenta la compra compulsiva de los consumidores, este campo se vuelve crítico para muchas empresas.

- Consideraciones medioambientales: la preocupación por el medioambiente se ha incrementado en los últimos años de manera notable a todos los niveles. Aunque no son un número representativo de publicaciones, debido a la relevancia de las aportaciones de los autores, se le ha dedicado un apartado propio en esta revisión.

Además, de acuerdo a la metodología aplicada, cada documento ha sido clasificado según su tipología: Estudios empíricos (EMP), revisiones bibliográficas (REV), modelos matemáticos (MOD), estudios de caso (CS) y modelos conceptuales (THE).

A modo resumen, la Tabla 1 clasifica todas las publicaciones del estudio en función tanto de su temática como del método de estudio aplicado. La Figura 1 complementa dicho resumen y muestra los resultados cuantitativos del reparto de las publicaciones de acuerdo con los 2 criterios descritos anteriormente.

Tabla 1 Resumen de los artículos analizados según su temática y metodología de estudio

\begin{tabular}{|c|c|c|c|c|c|}
\hline Grupo & EMP & MOD & CS & REV & THE \\
\hline $\begin{array}{l}\text { Adopción y diseño } \\
\text { del canal online }\end{array}$ & $\begin{array}{l}\text { Cui y Pan (2015), } \\
\text { Ghobakhloo et al. } \\
\text { (2011), Hübner et al. } \\
\text { (2015), Lin, W. T. et } \\
\text { al. (2016), Tolstoy et } \\
\text { al. (2016), Verbrak- } \\
\text { en et al. (2014), }\end{array}$ & $\begin{array}{l}\text { Yu et al. (2015), Lu y Liu } \\
\text { (2015), Li et al. (2015), } \\
\text { Xiong et al. (2012), } \\
\text { Zhang \& Zhang (2013), } \\
\text { Xu et al. (2012) }\end{array}$ & & $\begin{array}{l}\text { Aulkemeier et al. } \\
(2016), \quad \text { Stritto y } \\
\text { Schiraldi (2013) }\end{array}$ & \\
\hline Precios & Gümüs et al. (2013) & $\begin{array}{l}\text { Dan et al. (2014), Gao } \\
\text { et al. (2016), Li et al. } \\
\text { (2016), Zhang et al. } \\
\text { (2015), Lu y Liu (2013), } \\
\text { Sayadi y Makui (2014), } \\
\text { Peng et al. (2016) }\end{array}$ & & & \\
\hline
\end{tabular}




\begin{tabular}{|c|c|c|c|c|c|}
\hline Servicio al cliente & $\begin{array}{l}\text { Doherty et al. } \\
\text { (2015), Floh y Madl- } \\
\text { berger (2013), Rao } \\
\text { et al. (2011), Özpo- } \\
\text { lat y Jank (2015), } \\
\text { Wang et al. (2016), } \\
\text { Wen et al. (2014), } \\
\text { Xu et al. (2013) }\end{array}$ & & & Zuo et al. (2013) & $\begin{array}{l}\text { Otero et al. } \\
(2014)\end{array}$ \\
\hline E-fulfillment & $\begin{array}{l}\text { Hübner et al. (2016), } \\
\text { Lin, Y. et al. (2016), } \\
\text { Mahar et al. (2012) }\end{array}$ & $\begin{array}{l}\text { Cleophas y Ehmke } \\
\text { (2014), Henn (2012), } \\
\text { Hong et al. (2013), } \\
\text { Pérez-Rodríguez } \\
\text { (2015), Wang, X. et al. } \\
\text { (2014a), Subramanian } \\
\text { et al. (2015), Zhang et } \\
\text { al. (2016) }\end{array}$ & $\begin{array}{l}\text { Gallino y Moreno } \\
\text { (2014), Ghezzi et al. } \\
\text { (2012), Huang y Yin } \\
\text { (2014) }\end{array}$ & & $\begin{array}{l}\text { Izzah et al. } \\
(2016)\end{array}$ \\
\hline Gestión de stocks & $\begin{array}{l}\text { Peinkofer et al. } \\
(2015)\end{array}$ & $\begin{array}{l}\text { Bhatnagar y Syam } \\
\text { (2013), Chen et al. } \\
\text { (2011), Cheng et al. } \\
\text { (2016), Ma et al. (2016), } \\
\text { Mirzahosseinian et al. } \\
\text { (2011), Rui y Kaijun } \\
\text { (2013), Schneider y } \\
\text { Klabjan (2013), Cheong } \\
\text { et al. (2015), Wang, X. et } \\
\text { al. (2014b) }\end{array}$ & & & \\
\hline $\begin{array}{ll}\text { Devoluciones } & \text { y } \\
\text { logística inversa }\end{array}$ & $\begin{array}{l}\text { Rabinovich et al. } \\
\text { (2011), Ramanathan } \\
\text { (2011), Thiyagara- } \\
\text { jan y Ali (2016) }\end{array}$ & Liu (2014) & & & \\
\hline $\begin{array}{l}\text { Consideraciones } \\
\text { medioambientales }\end{array}$ & & Yan et al. (2016) & $\begin{array}{l}\text { Borggren et al. } \\
(2011)\end{array}$ & $\begin{array}{l}\text { Mangiaracina et al. } \\
(2015)\end{array}$ & \\
\hline
\end{tabular}
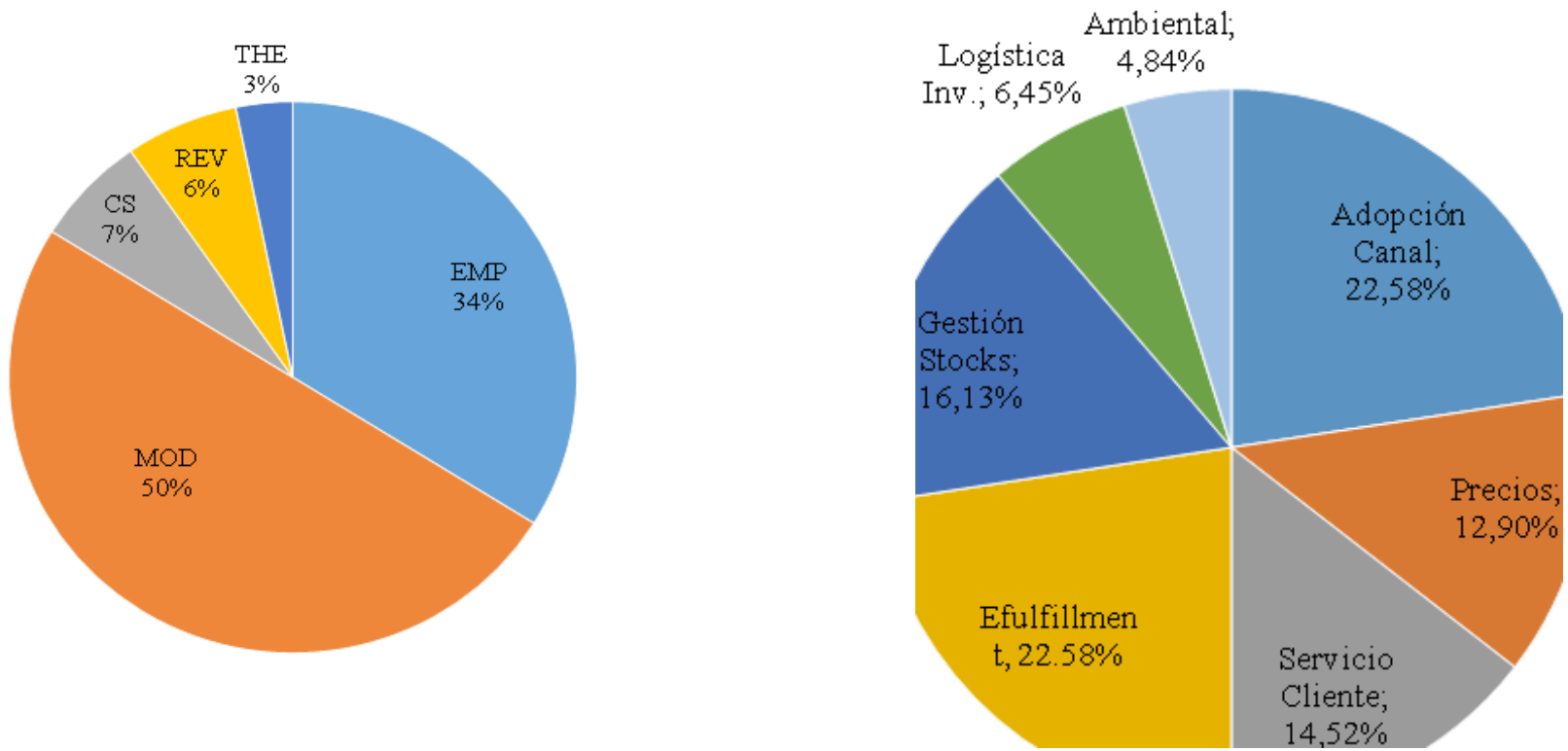

Figura 1 Distribución de los artículos analizados según temáti- 
Como se puede observar en los resultados obtenidos, la adopción y el diseño del canal y el e-fulfillment son las dos temáticas más abordadas en la literatura de los últimos años, alcanzando entre ambas cerca del $45 \%$ de las publicaciones totales. Les siguen la gestión de stocks, el servicio al cliente y el establecimiento de los precios con alrededor de entre un $15 \%$ y un $10 \%$ de las publicaciones cada una. Por último, la logística inversa y las consideraciones medioambientales son las dos temáticas menos abordadas, con apenas un $10 \%$ entre ambas.

De acuerdo con la metodología de investigación seguida por los autores, los modelos matemáticos y los estudios empíricas engloban prácticamente el total de las publicaciones (más del $80 \%$ entre ambas), mientras que de entre case-studies, revisiones bibliográficas y modelos teóricos, ninguno llega a alcanzar el $10 \%$. Los modelos matemáticos constituyen un $50 \%$ y son la metodología de investigación más común.

De manera conjunta, se observa que los modelos matemáticos predominan en áreas como el establecimiento de los precios, la gestión de stocks y el e-fulfillment, mientras que los modelos empíricos se centran en el análisis del servicio al cliente, en donde la mayoría de las publicaciones elaboran cuestionarios y/o entrevistas para analizar el comportamiento y preferencias de los clientes. La adaptación y diseño del canal es el único área en la que existe un equilibrio entre los modelos empíricos y los matemáticos. La mayoría de modelos empíricos en este ámbito describen los factores clave para adentrarse en el nuevo canal con éxito, mientras que los modelos matemáticos tratan de predecir el comportamiento de la cadena de suministro con la aparición de la figura del e-retailer.

\section{Discusión}

En esta sección, analizamos en detalle cada una de las publicaciones que se han incluido en la revisión bibliográfica en función de la temática en la que han sido categorizadas. Como se explicó anteriormente, algunas publicaciones pueden clasificarse en más de un área, y es por ello por lo que se citan en más de uno de los puntos descritos a continuación, siendo sus aportaciones relevantes en cada uno de ellos.

\section{Adopción y diseño del canal online}

Los factores clave en la adopción del e-commerce son analizados por Ghobakhloo et al. (2011), quiénes señalan como los más relevantes la perspectiva de obtener una ventaja competitiva y la compatibilidad con sus métodos actuales; otros aspectos, como el coste y la figura del CEO, tienen un papel relevante pero secundario. Por otra parte, la relevancia de las tecnologías de la información y las comunicaciones (TICs) en el canal on-line es especialmente significativa (Lin, W. T. et al., 2016; Xu et al., 2012). Aulkemeier et al (2016) confirman este hecho y revisan las tendencias en arquitectura del canal electrónico. Stritto y Schiraldi (2013) establecen un marco de referencia orientado a gestores tratando de alinear los intereses estratégicos con el diseño del canal de venta online, centrándose en todas las consideraciones logísticas que van a definir el diseño de la nueva cadena de suministro. Además, de acuerdo con el estudio de Verbraken et al. (2014) que predice el comportamiento online de los consumidores analizando datos de sus redes sociales, la tipología de producto es uno de estos elementos principales que influye en su aceptación en el canal online. En el mismo estudio se analiza también la aceptación del canal online según el perfil del cliente y las conexiones sociales con otros individuos, dando lugar a un modelo predictivo sobre la intención de compra.

Un aspecto que marca la diferencia notablemente entre ambos canales es la diferencia de coste entre uno y otro. Yu et al. (2015) demuestran que el coste de la venta online puede ser menor que el offline por causa de las diferencias entre los costes de transporte y procesamiento. Dicha reducción del coste ha animado a ciertos proveedores a integrarse verticalmente tratando de alcanzar el mercado minorista directamente, llegando a convertirse en competidores de sus propios clientes. Dicho efecto ha sido estudiado por $\mathrm{Li}$ et al. (2015), llegando a la conclusión de que tales "invasiones" dan lugar a un incremento de la presión competitiva y a una reducción del beneficio de la cadena de su-ministro, lo cual lleva a una situación de lose-lose entre el proveedor mayorista y el re-tailer. Esta casuística de competición entre canales lleva a cuestionarse la idoneidad del uso del canal "directo" u on-line según el tipo de empresa. Cui y Pan (2015) afirman que los conflictos entre canales son una de las principales causas de fracaso en la adopción del e-commerce. Como contraparte, Xiong et al. (2012), mediante una simulación, estudian los comportamientos de fabricantes y distribuidores para comprobar el impacto en la cadena de suministro de una apuesta del fabricante por el doble canal. Sus datos apuntan a que el distribuidor aumenta generalmente sus beneficios en este caso, a pesar de la irrupción del fabricante en su mercado. Una simulación similar es la realizada por Lu y Liu (2015), utilizando un modelo de teoría de juegos donde se tienen en cuenta 2 parámetros principales: la eficiencia del canal online y el grado de aceptación de dicho canal por los clientes. Usando su modelo, los beneficios de fabricante y minorista podr-ían predecirse antes de la 
entrada en un nuevo canal. Otro modelo, el de Xu et al. (2012), detalla como los fabricantes tienen la opción de utilizar el canal online, aunque a priori no les resulte especialmente rentable, para tener mayor control sobre la fijación de los precios de venta fijados por los retailers. Para lograr la deseable situación win-win entre fabricante y distribuidor, Li et al. (2016) proponen mecanismos de coordinación entre los mismos.

Como punto a favor de la adopción del canal online, Tolstoy et al. (2016) a través de un amplio estudio de cientos de compañías suecas del textil con presencia online, demuestran que la incorporación a este canal fomenta en gran medida la internacionalización de las ventas, tras comparar el ratio de empresas con presencia en el mercado web frente a otras que no la tienen.

En su análisis sobre las competencias necesarias en cada fase de la implantación del e-commerce, Cui y Pan (2015) determinan que la última fase de la implantación del comercio electrónico es la integración de los canales online y offline. En el caso de aquellas empresas que desarrollen una estrategia multicanal, será de vital importancia que las mismas traten de buscar sinergias entre ambos modelos de venta. Una de las aportaciones más importantes encontradas sobre la integración de los procesos logísticos en este entorno ha sido el análisis llevado a cabo por Hübner et al. (2015). En él, los autores identifican las principales operaciones a integrar entre ambos canales, en las que se incluyen: el diseño de la red de almacenes y flujos de materiales en la cadena de suministro, la gestión de stocks, la preparación de pedidos y la gestión de la capacidad logística. Para cada uno de estos elementos, los autores proponen diversas alternativas en la integración de canales y centralización de recursos. Muchas de estas aportaciones se verán en apartados concretos a lo largo de nuestra revisión.

\section{$\underline{\text { Precios }}$}

Con la llegada del comercio online, uno de las situaciones más comunes es que los fabricantes comiencen a vender sus productos directamente a los clientes, poniendo a los retailers tradicionales en posiciones de riesgo y alterando por completo la tradicional cadena de suministro (Gao et al., 2016; Zhang et al., 2015; Sayadi y Makui, 2014). Dan et al. (2014) sostiene que en estos casos los retailers tradicionales tienen distintas op-ciones como centrarse más en productos de alto precio que suelen funcionar mejor en el negocio tradicional, o incluso, si la sensibilidad de los consumidores por el canal tradicional se mantiene alta, la posibilidad de aumentar los precios de los productos para compensar la reducción en las ventas. Lu y Liu (2013) llegan a una conclusión distinta y establecen que el retailer ha de centrarse en mantener la aceptación del canal tradicional, pero manteniendo los precios lo más estables posibles, ya que precios diferenciadores favorecen las ventas del fabricante.

Por otro lado, cuando se buscan sinergias en este nuevo entorno, tanto retailers como fabricantes pueden beneficiarse. Peng et al. (2016) demostraron que las promociones de ventas llevadas a cabo por fabricante y retailer en las que ambos comparten el coste de las mismas pueden llegar a ser muy beneficiosas para ambos.

En el caso concreto de una Green Supply Chain, Li et al. (2016) analizaron las estrategias de precios y de posicionamiento 'green' en la SC para ver cómo afectan a los fabricantes y retailers. Por un lado, concluyeron que si el coste 'green' de un producto sobrepasa cierto umbral, incorporar el canal directo no es rentable para el fabricante. Por otro lado, cuando el coste 'green' aumenta y la sensibilidad 'green' del cliente decae, los precios tanto online como offline tienden a la baja.

Por último, Gümüs et al. (2013) incorporan consideraciones logísticas al establecimiento de los precios. Los autores elaboran un modelo de Teoría de Juegos y un análisis empírico de varios e-retailers con 2 productos (impresoras y cámaras), para sacar con-clusiones sobre cuando incluir los costes de transporte en el precio del producto, en función de la respuesta de los clientes y el tipo de producto.

\section{$\underline{\text { Servicio al cliente }}$}

Muchos factores afectan al servicio percibido por los clientes en el comercio electrónico, entre los que destacan la calidad de la web, la calidad en los procesos de transacción y, finalmente, la calidad propia del producto (Wen et al., 2014; Floh y Madberger, 2013). Sobre la plataforma web, uno de los temas más críticos para un negocio online, Zuo et al. (2013), desde una perspectiva de QFD, obtienen los factores más relevantes del servicio de la interfaz y usabilidad de la plataforma web. A su vez, Otero et al. (2014) muestra los factores clave que han de tener en cuenta las SMEs cuando desarrollan su web de venta online desde un punto de vista muy práctico. Con una muestra amplia de casi 500 e-retailers estadounidenses, Özpolat y Jank (2015), afirman que los sellos de calidad otorgados por terceros tienen un impacto mayor en empresas pequeñas que en aquellas más grandes en la venta online. Estos sellos además presentan un mayor impacto conforme el valor de producto aumenta. 
Por otro lado, cada vez existe una interrelación mayor entre el área del marketing y de la logística. Desde la llegada del comercio electrónico, los clientes compran cada vez más, no sólo un producto físico, sino también un servicio de entrega personalizado (plazo, punto de recogida, etc.). Wang et al. (2016) establece 6 factores clave distintos: quién realiza el envío (el propio e-retailer o un tercero), la calidad del producto enviado, la calidad del servicio de entrega por parte del personal, la información provista al cliente sobre el envío, el tiempo de envío y la flexibilidad en la entrega. Estos factores y otros son clasificados en 2 grandes grupos por $\mathrm{Xu}$ et al. (2013), que recalca la diferencia entre aquellos que se centran en la eficacia del servicio, entendida como que el producto adecuado llegue en perfectas condiciones y en el tiempo prometido; y aquellos que afectan al proceso de envío en sí mismo, como pueden ser la flexibilidad, el seguimiento del pedido o la actitud del personal que entrega el pedido. En su estudio demuestra que aquellos factores ligados al proceso, y que hasta la fecha carecían de relevancia, son cada vez más importantes para los consumidores. Esta parte logística tiene del servicio tiene 4 pilares principales según Ghezzi et al. (2012): tiempo de ciclo, puntualidad, flexibilidad y gestión de devoluciones. Por otro lado, Rao et al. (2011) realizaron también un estudio empírico en el que se obtuvo como resultado que la calidad del proceso de distribución física (midiendo la calidad percibida por el cliente a través de 4 elementos: variedad de opciones, disponibilidad de productos, fiabilidad del seguimiento del envío y puntualidad en la entrega) era el factor más influyente en relación con la satisfacción y la retención del cliente.

Finalmente, mediante un estudio empírico basado en más de 225 cuestionarios a managers de empresas e-retailers de UK, Doherty et al. (2015), establecen el servicio postventa ofrecido a los consumidores online como uno de los factores más importantes de la venta online, y al mismo tiempo uno de los menos desarrollados por los e-retailers.

\section{E-fulfillment}

El e-fulfillment se entiende como aquel conjunto de procesos necesarios para hacer llegar el producto al cliente desde que se produce la venta, por lo que comúnmente la literatura lo entiende como las operaciones de preparación de pedidos, el envío al cliente y la venta misma. La entrega final al cliente en el e-commerce se ha conocido ampliamente como last mile delivery, y es un área crítica debido a la necesidad de llegar a múltiples destinos con numerosos productos distintos. Los modelos multicanal aportan a esta casuística nuevas opciones, al disponer de tiendas físicas que pueden actuar como puntos de recogida para el cliente o como simples almacenes que ayuden a optimizar los flujos de transporte.

Entre estos aspectos, la subcontratación de los servicios de last mile delivery ha sido objeto de estudio recurrentemente. Teniendo en cuenta que lo más habitual en el negocio online es la delegación de los servicios de transporte a un operador logístico, conocidos como third-party logistics provider o 3PL, muchos de las estudios publicados estudian los factores que influyen en la decisión de subcontratar o no estos servicios (Huang y Yin, 2014; Izzah, 2016; Lin, Y. et al., 2016; Ghezzi et al., 2012). De entre ellos, destacamos el trabajo de Ghezzi et al. (2012), que han recopilado y analizado datos de 28 empresas de diferentes sectores de Italia que realizan ventas online, definiendo las diferentes estrategias logísticas posibles (subcontratación $100 \%$, subcontratación parcial, no subcontratación) en función del tipo de producto en venta. Según sus resultados, para productos de alto valor y un servicio logístico esperado bajo (como productos de informática o electrodomésticos) la externalización de los servicios de envío a un 3PL resulta la mejor opción. Lo opuesto ocurre cuando el valor del producto es bajo y por el contrario la logística es clave (por ejemplo en productos alimenticios), en donde el con-trol ha de recaer al $100 \%$ en el e-retailer. En caso de que un e-retailer se decida por la externalización, Subramanian et al. (2015) aporta un análisis de gran utilidad sobre qué factores tener en cuenta para evaluar a los distintos $3 \mathrm{PL}$, entre los que resulta tener la mayor importancia de todas la flexibilidad del proveedor de servicios logísticos.

Otro grupo de publicaciones relacionadas con el transporte está representado por modelos de optimización. Cleophas y Ehmke (2014) muestran una manera de optimizar los envíos realizados en zonas urbanas basándose en dos fundamentos básicos: la capacidad de distribución y la demanda esperada. En base a esta demanda esperada se crean rutas que minimizan el coste total de la distribución, para posteriormente aceptar o rechazar los envíos reales en función de su urgencia y tratando de minimizar el desvío en el coste previsto. Hong et al. (2013) desarrollan un modelo algorítmico de rutas para optimizar la distribución de los productos desde un almacén central a diversos clientes.

En la gestión multicanal, la relación entre las tiendas físicas y tiendas online es de vital importancia para aquellas empresas con presencia en el mercado tradicional y que se introducen en el nuevo modelo. Esta casuística ha sido ampliamente estudiada de forma empírica por Gallino y Moreno (2014). En este artículo, se analiza el comportamiento de las ventas tras la 
implantación de "recogida en tienda" (pick-up at store) para los productos de la web. El autor halla evidencias empíricas de que el uso de pick-up points tiene un efecto ligeramente negativo sobre las ventas de la tienda online (el autor achaca esto al efecto ROPO "Research Online, Purchase Offline"). Sin embargo, también encuentra evidencias empíricas de que la implantación de los pick-up points en una tienda física tiene un efecto muy positivo en las ventas de la tienda física (fomenta la venta cruzada). Por lo tanto, el efecto global es positivo, lo cual alienta a las empresas que dispongan tanto de tiendas físicas como online a que implanten este tipo de recogidas en tienda.

Hübner et al. (2016) define una estructura muy completa con las diferentes variantes posibles que tiene un retailer para llevar un producto online al cliente en caso de una empresa con gestión multicanal, combinando variables como el punto de envío (a casa o recogida en tienda), el tiempo de envío prometido o el área geográfica donde opera la empresa. Otro estudio, el de Mahar et al. (2012), sostiene que las empresas con tienda física que ofrecen la posibilidad de recogida en tienda pueden reducir el coste logístico total (transporte, almacenamiento, rotura...) ofreciendo sólo ciertas tiendas como puntos de recogida posibles al consumidor, y que serán las más óptimas a nivel de coste. Estas relaciones entre la recogida en tienda, la recogida en "pick-up point" externo y la entrega directa han sido también modelizadas por Wang, X. et al. (2014a).

Otro grupo de publicaciones que engloba el e-fulfillment tiene que ver con la gestión de las operaciones que se llevan a cabo en los centros de almacenaje. En concreto, el tema más abordado es la preparación de los pedidos o picking. Hübner et al. (2015), tras un estudio que incluía a más de 33 retailers líderes europeos con presencia online y offline, concluyeron que en el $95 \%$ de los mismos los costes de picking para la venta online eran superiores a los de la venta en tienda, lo cual era debido a que alrededor del $50 \%$ de los pedidos online sólo incluían de media entre 1 y 2 productos. La técnica más destacable en el picking online es la agrupación de pedidos o batching. (Henn, 2012; Pérez-Rodríguez, 2015; Zhang et al., 2016). El modelo de Henn (2012) tiene gran relevancia por las numerosas alternativas que presenta. El autor propone un cierto nivel de agrupa-ción de pedidos y unas rutas determinadas de recogida de productos, y todo ello en un ambiente de demanda variable. El modelo por un lado optimiza los procesos de recogida, por lo que ayuda a aumentar el ratio global de pedidos en plazo y reducir el lead-time de cada uno de ellos. Por otro, la nivelación de la demanda ayuda a evitar picos de trabajo, que a su vez reducen la necesidad de plantilla extra y con ello el coste global de las operaciones.

\section{Gestión de stocks}

Es uno de los elementos más estudiados, principalmente en la logística multicanal. La visión principal de la mayoría de los autores es que el canal online bien gestionado es un complemento y no una competencia para la venta física en las tiendas (Bhatnagar y Syam, 2013; Ma et al., 2016). La importancia de una adecuada gestión de stocks desde el punto de vista logístico-comercial se define perfectamente en los dos estudios elaborados por Peinkofer et al. (2015), en los cuales se analiza el impacto en la satisfacción de los clientes que tienen las roturas de inventario en la venta online. La aportación más importante por parte de los autores es el descubrimiento de que las roturas de stock tienen un impacto menor sobre la retención de clientes cuando van ligadas a promociones especiales de precios, lo que puede permitir a las empresas ajustar sus stocks a la baja en estos casos.

Bhatnagar y Syam (2013) desarrollan un modelo que optimiza la asignación de los stocks a uno u otro canal, aunque sin contemplar opciones como el pick-up at store, y concluyendo que ciertos productos con altos costes de almacenaje y transporte pueden llegar a ser retirados de las tiendas físicas y vendidos únicamente online dependiendo de la elasticidad de la demanda de los clientes respecto a uno u otro canal. Otros modelos de optimización integran más la venta multicanal a nivel de stocks (Schneider y Klabjan, 2013; Mirzahosseinian et al., 2011) y describen modelos que estandarizan el sistema de reaprovisionamiento desde los almacenes centrales hacia las tiendas y los niveles stocks en ambos puntos. Por otro lado, Rui y Kaijun (2013) diseña un modelo de gestión basado en TOC (Theory of Constrains) que optimiza la cantidad de inventario que se debe 'reservar' en los almacenes centrales para cubrir las necesidades del canal de venta online.

Finalmente, existe una importante aportación en la literatura sobre la gestión de stocks en condiciones de drop-shipping, término con el que se conoce a la técnica de gestión de la cadena de suministro en la que los e-retailers no almacenan stocks de ciertos productos en venta, sino que circulan los pedidos directamente a sus proveedores y son es-tos los que gestionan directamente el envío de esos productos a los clientes. Wang, X. et al. (2014b), Cheng et al. (2016) y Chen et al. (2011) presentan modelos de optimización para que el e-retailer pueda saber en qué casos es rentable utilizar la técnica de drop-shipping, y, de hacerlo, los niveles de inventario que han de mantener tanto él como sus proveedores. Por último, Cheong et al. (2015) nos describe los problemas que presenta una mala compartición de la información entre e-retailers 
y proveedores sobre los stocks en condiciones de drop-shipping.

\section{Devoluciones y logística inversa.}

El impacto de una adecuada gestión de las devoluciones en la retención de clientes fue estudiada empíricamente por Ramanathan (2011). En un estudio que incluía a más de 1.000 tiendas online, el estudio concluye que los retornos son más críticos para los clientes en el caso de la venta de productos considerados de alto riesgo (precio alto y ambigüedad en las especificaciones) y en aquellos de muy bajo riesgo (bajo precio y baja ambigüedad en las especificaciones), siendo menos crítico en el resto de productos. Las devoluciones de los productos online también fueron analizados por Rabinovich et al. (2011), siendo el precio de los productos y el tamaño las dos principales variables influyentes. La conclusión más importante del estudio es que las devoluciones se concentran mayormente en un reducido número de productos que no consiguen cubrir las ex-pectativas de los consumidores por diversas razones, y que son aquellos productos sobre los que los retailers han de actuar.

En gestión de devoluciones, se pueden encontrar hasta 3 tipos de instalaciones según la etapa del proceso: puntos de recolección, centros de reciclado y centros de reprocesado. Liu (2014), a través del diseño de un algoritmo genético, es capaz de aproximar la loca-lización óptima de cada uno de estos puntos de la cadena de suministro de ciclo cerrado closed-loop supply chain. Por último, un estudio empírico elaborado por Thiyagarajan y Ali (2016) a empresas de 4 sectores clave del retailing (calzado, textil, mueblería y electrodomésticos) determina la dificultad que tiene para los retailers online la gestión de la logística inversa. Además, también enumera las principales barreras que dificultan la implantación de un correcto sistema de gestión de retornos y las clasifica en 4 categorías: barreras culturales, barreras estratégicas, barreras tecnológicas y barreras externas.

\section{Consideraciones medioambientales}

En concreto en el comercio electrónico, pueden apreciarse 4 grandes asuntos que captan el interés de la investigación sobre el medio ambiente según Mangiaracina et al. (2015): el diseño de la red de distribución, el impacto medioambiental del transporte, el almacenamiento y el packaging.

El transporte es una de las actividades que mayor impacto medioambiental ocasionan, en particular el reparto de última milla o last mile delivery. En el caso del e-commerce, la preocupación por un aumento de este impacto por el hecho de realizar envíos de volúmenes cada vez menores y cliente por cliente, está presente en la literatura y ha sido detalladamente estudiado en la revisión bibliográfica llevada a cabo por Mangiaracina et al. (2015). Los autores identifican prácticas concretas que pueden ser emprendidas con el fin de reducir las emisiones de gases de efecto invernadero: usar vehículos de combustibles alternativos, con un impacto ambiental significativamente menor, renovar la flota por una con nuevas tecnologías menos contaminantes y tratar de realizar envíos mayores, consolidando pedidos en la medida de los posible. Las ventajas de este tipo de colaboraciones, han sido también estudiadas a nivel de coste por Yan et al. (2016), quien obtiene como resultado que a mayor colaboración los beneficios para las partes involucradas aumentan.

Por otro lado, comparando el impacto ambiental de la venta online y la venta en tienda física, hay que tener en cuenta la cantidad de ítems que nunca serán vendidos que llegan a las tiendas, y que a la larga se convierten en obsoletos. Este dato puede variar am-pliamente de unos sectores a otros, por ejemplo, Ghezzi et al. (2012) mencionan que en el caso de libros y DVDs más de un 35\% de los artículos que llegan a las estanterías no se llegarán a vender.

Respecto al diseño de la red de distribución, las principales investigaciones tienen que ver con la evaluación del impacto ambiental de los envíos directos que fomenta, a priori, el comercio electrónico. Zhang \& Zhang (2013) compara 2 sistemas de distribución distintos (envío directo vs pick-up points) utilizando datos reales de una compañía de transporte en China. Sus resultados llevan a concluir que los pick-up points tienen un menor impacto ambiental, siendo la principal causa el hecho de que en los pick-up points no se producen apenas entregas fallidas, mientras que en los envíos directos hasta un $25 \%$ de las entregas según sus datos fueron fallidas, ocasionando viajes adicionales y la consecuente pérdida de eficiencia. Sin embargo, otros estudios que tienen en cuenta el impacto ambiental de los desplazamientos del cliente, obtienen resultados diferentes. Según Borggren et al. (2011), el ahorro de los pick-up points se produce solo si el cliente acude al mismo andando, ya que el conjunto de emisiones de todos los particulares que acuden al punto de recogida es generalmente mayor que la entrega a domicilio. Por ello, el envío directo al domicilio del cliente reduce las emisiones de gases de efecto in-vernadero.

Las actividades de almacén (almacenamiento y preparación de pedidos) y el packaging tienen un impacto significativo en el e-commerce. Los efectos de las actividades de almacén sobre el medioambiente cobran cierto protagonismo por la gran cantidad de recursos 
destinados al picking y a la preparación individual de pedidos. De un modo similar, el packaging para e-commerce tiene un impacto también notable, ya que, al contrario que los envíos a tiendas convencionales, requiere mucho más material para el envío a domicilio para garantizar la protección del producto, que típicamente se empaqueta individualmente. Debido a esto, Mangiaracina et al. (2015) determina que, en general, la implantación del comercio electrónico empeora el rendimiento medioambiental en estos dos aspectos.

\section{Conclusiones}

El objetivo principal de este artículo era ofrecer una recopilación bibliográfica actuali-zada sobre la nueva gestión de la cadena de suministro, con una visión centrada en la figura de los e-retailers. La adopción y el diseño del canal y el e-fulfillment han sido las dos temáticas más presentes en la literatura de los últimos años. Respecto a la adopción del canal online, numerosas publicaciones se han centrado en los factores que pueden hacer exitosa la adopción de este canal y las consecuencias de su adopción en la cadena de suministro tradicional. Sin embargo, quizás se eche en falta un conjunto de publicaciones que presenten a las empresas los pasos a seguir y las consideraciones que han de tener en cuenta para emprender su aventura online de un modo más práctico. El otro gran grupo lo constituye la literatura del e-fulfillment, que estudia ampliamente el transporte final al cliente y los nuevos modelos de venta como el pick-up at store. Por otro lado, áreas como la gestión de stocks y el establecimiento de los precios también tienen una presencia importante en la literatura, principalmente en forma de modelos matemáticos de optimización. El servicio al cliente, otra de las áreas más ampliamente analizadas, se centra sobre todo en hallar los factores clave que condicionan el comportamiento de los consumidores en el mercado online. La logística inversa y las consideraciones medioambientales se muestran como las 2 otras áreas descritas en nuestro análisis. En la primera, los autores se centran en describir los factores que afectan a las devoluciones y no tanto en cómo desarrollar un proceso interno de logística inversa eficiente. Las consideraciones medioambientales recaen principalmente en comparativas sobre el impacto ambiental de la venta online y la venta tradicional en tiendas.

Coincidiendo con Mangiaracina et al. (2015), creemos que existe una carencia de contenido en ciertos ámbitos logísticos, tanto operacionales como estratégicos. Por un lado, la alineación con proveedores al introducirse en el canal de venta online es uno de esos campos inexplorados y que bajo la opinión de los autores tiene una relevancia muy importante. En este sentido, únicamente el dropshipping aparece como una nueva técnica de gestión de proveedores. Pese a ello, las publicaciones analizadas se centraban única-mente en la gestión de stocks. Otras decisiones sobre el transporte inbound, los aprovisionamientos, la redefinición de SKUs (las unidades de ventas en los canales online y offline pueden variar mucho), cambios en la frecuencia de las entregas o la necesidad de búsqueda de nuevos proveedores al adentrarse en el canal de venta online no tienen prácticamente mención en toda la literatura analizada. La gestión de la capacidad logística, analizada por Hübner et al. (2015), es otro punto de gran interés y que requiere nuevos estudios que enseñen a las empresas cómo mejorarla, ya que se aprecian grandes diferencias entre el comportamiento de las demandas de los canales online y offline, y por lo tanto existe una nueva necesidad de satisfacer ambas con los menores recursos posibles. Por último, el packaging, como elemento diferenciador en las ventas online, pero también como elemento logístico clave para la optimización de los procesos logísticos (García-Arca et al. 2014), es otro de las áreas que no se han visto reflejadas en esta revisión bibliográfica.

\section{$6 \quad$ Referencias}

A.T. KEARNEY (2015). «Global Retail E-Commerce Keeps On Clicking». Extraído el 1 de Junio, 2016, de https://www.atkearney.com/ documents/10192/5691153/Global+Retail+E-Commerce+Keeps+On+Clicking.pdf/abe38776-266947ba-9387-5d1653e40409

AULKEMEIER, F., SCHRAMM, M., IACOB, M. E., y VAN HILLEGERSBERG, J. (2016). «A service-oriented e-commerce reference architecture». Journal of theo-retical and applied electronic commerce research, 11(1), pp. 26-45.

BHATNAGAR, A., y SYAM, S. S. (2014). "Allocating a hybrid retailer's assortment across retail stores: Bricks-and-mortar vs online». Journal of Business Research, 67 (6), pp. 1293-1302.

BORGGREN, C., MOBERG, A. y FINNVREDEN, G. (2011), «Books from an envi-ronmental perspective -part 1: environmental impacts of pa-per books sold in tradi-tional and internet bookshops». The International Journal of Life Cycle Assessment, Vol. 11 No. 2, pp. 138-147.

CLEOPHAS, C., y EHMKE, J. F. (2014). «When Are Deliveries Profitable? ॥. Business \& Information Systems Engineering, 6 (3), pp. 153-163. 
CUI, M., y PAN, S. L. (2015). «Developing focal capabilities for e-commerce adoption: A resource orchestration perspective». Information \& Management, 52 (2), pp. 200-209.

CUI, M., y PAN, S. L. (2015). «Developing focal capabilities for e-commerce adoption: A resource orchestration perspective». Information \& Management, 52 (2), pp. 200-209.

CHEN, J., CHEN, Y., PARLAR, M., y XIAO, Y. (2011). «Optimal inventory and ad-mission policies for drop-shipping retailers serving in-store and online customers». IIE Transactions, 43 (5), pp. 332-347.

CHENG, Y., LI, B., y JIANG, Y. (2016). «Optimal Choices for the E-Tailer with In-ventory Rationing, Hybrid Channel Strategies, and Service Level Constraint under Multiperiod Environments». Mathematical Problems in Engineering, 2016.

CHEONG, T., GOH, M., y SONG, S. H. (2015). «Effect of Inventory Information Dis-crepancy in a Drop $\square$ Shipping Supply Chain». Decision Sciences, 46 (1), pp. 193-213.

DAN, B., QU, Z. J., LIU, C., ZHANG, X. M., y ZHANG, H. Y. (2014). «Price and service competition in the supply chain with both pure play internet and strong bricks-and-mortar retailers». Journal of applied research and technology, 12 (2), pp. 212-222.

DOHERTY, N. F., SHAKUR, M., y ELLIS-CHADWICK, F. (2015). «The role of e-service quality management in the delivery business value». Journal of Retailing and Consumer Services, 27, pp. 52-62.

FLOH, A., y MADLBERGER, M. (2013). «The role of atmospheric cues in online im-pulse-buying behavior». Electronic Commerce Research and Applications, 12 (6), pp. 425-439.

GALLINO, S., y MORENO, A. (2014). «Integration of online and offline channels in retail: The impact of sharing reliable inventory availability information». Management Science, 60 (6), pp. 1434-1451.

GAO, J., WANG, X., YANG, Q., y ZHONG, Q. (2016). «Pricing Decisions of a Dual-Channel Closed-Loop Supply Chain under Uncertain Demand of Indirect Chan-nel». Mathematical Problems in Engineering, 2016.

GARCÍA-ARCA, J., PRADO-PRADO, J. C., \& GONZALEZ-PORTELA GARRIDO, A. T. (2014). «"Packaging logistics": promoting sustainable efficiency in supply chains». International Journal of Physical Distribution \& Logistics Management, 44(4), pp. 325-346.

GHEZZI, A., MANGIARACINA, R., y PEREGO, A. (2012). "Shaping the e-commerce logistics strategy: a decision framework». International Journal of En-gineering Business Management, 4.

GHOBAKHLOO, M., ARIAS-ARANDA, D., y BENITEZ-AMADO, J. (2011). «Adoption of e-commerce applications in SMEs». Industrial Management \& Data Systems, 111 (8), pp. 1238-1269.

GÜMÜŞ, M., LI, S., OH, W., y RAY, S. (2013). «Shipping fees or ship-ping free? A tale of two price partitioning strategies in online retailing». Production and Operations Management, 22 (4), pp. 758-776.

HENN, S. (2012). «Algorithms for on-line order batching in an order picking ware-house». Computers \& Operations Research, 39 (11), pp. 2549-2563.

HONG, X., JINGJING, Q., y XINGLI, T. (2013). «B2C E-commerce Vehicle Delivery Model and Simulation». Information Technology Journal, 12 (20), p. 5891.

HUANG, Y., y YIN, K. (2014). «Research on the Evaluation and Selection of Third-Party Logistics Providers in B2C E-Commerce Mode». Journal of Electronic Commerce in Organizations (JECO), 12 (2), pp. 74-88.

HÜBNER, A., HOLZAPFEL, A., y KUHN, H. (2015). «Operations management in multichannel retailing: an exploratory study». Operations Management Research, 8 (3-4), pp. 84-100.

HÜBNER, A., KUHN, H., y WOLLENBURG, J. (2016). "Last mile fulfilment and distribution in omnichannel grocery retailing: A strategic planning framework». International Journal of Retail \& Distribution Management, 44 (3), pp. 228-247.

HUNTER, M. G. (ED.). (2015). «Strategic Utilization of Information Systems in Small Business». IG/ Global.

IZZAH, N., RIFAI, D., y YAO, L. (2016). «Relationship-Courier Partner Logistics and E-Commerce Enterprises in Malaysia: A Review». Indian Journal of Science and Technology, 9 (9).

JIE, Y. U., SUBRAMANIAN, N., NING, K., y EDWARDS, D. (2015). «Product delivery service provider selection and customer satisfaction in the era of internet 
of things: a Chinese e-retailers' perspective». International Journal of Production Economics, 159, pp. 104-116.

LI, B., ZHU, M., JIANG, Y., y LI, Z. (2016). «Pricing policies of a competitive dual-channel green supply chain». Journal of Cleaner Production, 112, pp. 2029-2042.

LI, T., XIE, J., y ZHAO, X. (2015). «Supplier encroachment in competitive supply chains». International Journal of Production Economics, 165, pp. 120-131.

LIN, W. T., CHEN, Y. H., y CHOU, C. C. (2016). «Assessing the values of EC and IT separately and simultaneously and inputs substitution and complement: The CES-based stochastic frontier approaches». Information \& Management, 53 (1), pp. 109-121.

LIN, Y., LUO, J., CAI, S., MA, S., y RONG, K. (2016). «Exploring the service quality in the e-commerce context: a triadic view». Industrial Management \& Data Systems, 116 (3), pp. 388-415.

LIU, D. (2014). «Network site optimization of reverse logistics for E-commerce based on genetic algorithm». Neural Computing and Applications, 25 (1), pp. 67-71.

LU, Q., y LIU, N. (2013). «Pricing games of mixed conventional and e-commerce dis-tribution channels». Computers \& Industrial Engineering, 64 (1), pp. 122132.

LU, Q., y LIU, N. (2015). «Effects of e-commerce channel entry in a two-echelon sup-ply chain: A comparative analysis of single-and du-al-channel distribution systems». International Journal of Production Economics, 165, pp. 100-111.

MA, S., LIN, J., y ZHAO, X. (2016). «Online store discount strategy in the presence of consumer loss aversion». International Journal of Production Economics, 171, pp. 1-7.

MAHAR, S., SALZARULO, P. A., y WRIGHT, P. D. (2012). «Using online pickup site inclusion policies to manage demand in retail/E-tail organizations». Computers \& Operations Research, 39 (5), pp. 991999.

MANGIARACINA, R., MARCHET, G., PEROTTI, S., y TUMINO, A. (2015). «A re-view of the environmental implications of B2C e-commerce: a logistics perspec-tive». International Journal of Physical Distri- bution \& Logistics Management, 45 (6), pp. 565-591.

MIRZAHOSSEINIAN, H., MAKUI, A., TEIMOURY, E., RANJBAR-BOURANI, M., y AMOOZAD-KHALILI, H. (2011). «Impact of transportation system on total cost in a two-echelon dual channel supply chain». International Journal of Engineering, Transactions B: Applications, 24, pp. 27-36.

ÖZPOLAT, K., y JANK, W. (2015). «Getting the most out of third party trust seals: An empirical analysis». Decision Support Systems, 73, pp. 47-56.

PEINKOFER, S. T., ESPER, T. L., SMITH, R. J., y WILLIAMS, B. D. (2015). «As-sessing the Impact of Price Promotions on Consumer Response to Online Stockouts». Journal of Business Logistics, 36 (3), pp. 260-272.

PENG, G., TIAN, X., y CHEN, Q. (2016). «Online Cooperative Promotion and Cost Sharing Policy under Supply Chain Competition». Mathematical Problems in En-gineering, 2016.

PÉREZ-RODRÍGUEZ, R., HERNÁNDEZ-AGUIRRE, A., y JÖNS, S. (2015). "A continuous estimation of distribution algorithm for the online order-batching problem». The International Journal of Advanced Manufacturing Technology, 79 (1-4), pp. 569-588.

RABINOVICH, E., SINHA, R., y LASETER, T. (2011). "Unlimited shelf space in In-ternet supply chains: Treasure trove or wasteland?». Journal of Operations Man-agement, 29 (4), pp. 305-317.

RAMANATHAN, R. (2011). "An empirical analysis on the influence of risk on rela-tionships between handling of product returns and customer loyalty in E-commerce». International Journal of Production Economics, 130(2), pp. 255-261.

RAO, S., GOLDSBY, T. J., GRIFFIS, S. E., y IYENGAR, D. (2011). «Electronic Lo-gistics Service Quality (e $\square$ LSQ): Its Impact on the Customer's Purchase Satisfaction and Retention». Journal of Business Logistics, 32(2), pp. 167-179.

RUI, S., y KAIJUN, L. (2013). «Inventory Control Policy for E-tail Organizations Based on TOC». Information Technology Journal, 12 (24), p. 8171.

SAYADI, M. K., y MAKUI, A. (2014). «Optimal advertising decisions for promoting retail and online channels in a dynamic framework». International Transactions in Operational Research, 21 (5), pp. 777-796. 
SCHNEIDER, F., y KLABJAN, D. (2013). «Inventory control in multi-channel retail». European Journal of Operational Research, 227 (1), pp. 101-111.

SETIA, P., y PATEL, P. C. (2013). "How information systems help create OM capa-bilities: Consequents and antecedents of operational absorptive capacity». Journal of Operations Management, 31 (6), pp. 409-431.

SEURING, S., y MÜLLER, M. (2008). «From a literature review to a conceptual framework for sustainable supply chain management». Journal of cleaner production, 16 (15), pp. 1699-1710.

STRITTO, G. D., y SCHIRALDI, M. M. (2013). «A Strategy Oriented Framework for Food and Beverage E-Supply Chain Management». International Journal of Engi-neering Business Management, 5.

THIYAGARAJAN, G., y ALI, S. (2016). «Analysis of Reverse Logistics Implementa-tion Barriers in Online Retail Industry». Indian Journal of Science and Technology, 9 (19).

TOLSTOY, D., JONSSON, A., y SHARMA, D. D. (2016). «The Influence of a Retail Firm's Geographic Scope of Operations on Its International Online Sales». Interna-tional Journal of Electronic Commerce, 20 (3), pp. 293-318.

VERBRAKEN, T., GOETHALS, F., VERBEKE, W., y BAESENS, B. (2014). "Pre-dicting online channel acceptance with social network data». Decision Support Sys-tems, 63, pp. 104-114.

WAHBA, P. (2015, Noviembre 6). "This Chart Shows Just How Dominant Amazon Is». Fortune. Extraído el 18 de Mayo, 2016, de http://fortune.com/2015/11/06/ amazon-retailers-ecommerce/

WANG, C., MAO, Z., O'KANE, J., y WANG, J. (2016). «An exploration on e-retailers' home delivery-strategic elements and their prioritisation». Business Process Man-agement Journal, 22 (3).

WANG, X., WU, Y., y LIANG, L. (2014 b). «A New Business Mode for FTs Chain in an E-Commerce Environment». Mathematical Problems in Engineering, 2014.

WANG, X., ZHAN, L., RUAN, J., y ZHANG, J. (2014a). "How to Choose "Last Mile" Delivery Modes for E-Fulfillment». Mathematical Problems in Engineering, 2014.a
WEN, C., R. PRYBUTOK, V., BLANKSON, C., y FANG, J. (2014). «The role of E-quality within the consumer decision making process». International Journal of Operations \& Production Management, 34 (12), pp. 1506-1536.

XIONG, Y., YAN, W., FERNANDES, K., XIONG, Z. K., y GUO, N. (2012). "BBicks vs. Clicks": The impact of manufacturer encroachment with a dealer leasing and selling of durable goods». European Journal of Operational Research, 217 (1), pp. 75-83.

XU, H., LIU, Z. Z., y ZHANG, S. H. (2012). «A strategic analysis of dual-channel sup-ply chain design with price and delivery lead time considerations». International Journal of Production Economics, 139 (2), pp. 654-663.

XU, J., JIANG, L., y LI, Y. (2013). «Service requirement for terminal delivery: An em-pirical study from the perspective of online shoppers». Journal of Industrial Engi-neering and Management, 6 (4), pp. 12231237.

YAN, R., PEI, Z., y MYERS, C. (2016). «Do channel members value the multiple-cooperation strategy?» Journal of Retailing and Consumer Services, 30, pp. 84-95.

YU, Y., HAN, X., LIU, J., y CHENG, Q. (2015). «Supply chain equilibrium among companies with offline and online selling channels». International Journal of Pro-duction Research, 53 (22), pp. 6672-6688.

ZHANG, J., WANG, X., y HUANG, K. (2016). «Integrated on-line scheduling of order batching and delivery under B2C e-commerce». Computers \& Industrial Engineering, 94, pp. 280-289.

ZHANG, L., y ZHANG, Y. (2013). «A Comparative Study of Environmental Impacts of Two Delivery Systems in the Business-to-Customer Book Retail Sector». Journal of Industrial Ecology, 17(3), pp. 407-417.

ZHANG, R., MI, J. J., y LIU, B. (2015). «Pricing Decisions of Competing Tobacco En-terprises with Online Channel». Discrete Dynamics in Nature and Society, 2015.

ZUO, W., HUANG, Q., FAN, C., y ZHANG, Z. (2013). «Quality management of B2C e-commerce service based on human factors engineering». Electronic Commerce Research and Applications, 12 (5), pp. 309-320. 